\title{
Large scale inkjet-printing of carbon nanotubes electrodes for antioxidant assays in blood bags
}

\author{
Andreas Lesch ${ }^{\mathrm{a}}$, Fernando Cortés-Salazar ${ }^{\mathrm{a}}$, Michel Prudent ${ }^{\mathrm{b}}$, Julien Delobel ${ }^{\mathrm{b}}$, Shokoufeh Rastgar $^{\mathrm{c}}$, \\ Niels Lion ${ }^{\mathrm{b}}$, Jean-Daniel Tissot ${ }^{\mathrm{b}}$, Philippe Tacchini ${ }^{\mathrm{d}}$, Hubert H. Girault ${ }^{\mathrm{a}, *}$ \\ a Ecole Polytechnique Fédérale de Lausanne, Laboratoire d'Electrochimie Physique et Analytique, Station 6, CH-1015 Lausanne, Switzerland \\ ${ }^{\mathrm{b}}$ Service Régional Vaudois de Transfusion Sanguine, Unité de Recherche et Développement, CH-1066 Epalinges, Switzerland \\ ${ }^{\mathrm{c}}$ Department of Chemistry, Sharif University of Technology, Tehran 11155-9516, Iran \\ ${ }^{\mathrm{d}}$ Edel for Life, PSE-B/EPFL, CH-1015 Lausanne, Switzerland
}

\section{A R T I C L E I N F O}

\section{Article history:}

Received 7 December 2013

Received in revised form 19 December 2013

Accepted 20 December 2013

Available online 8 January 2014

\section{Keywords:}

Carbon nanotubes

Amperometric sensors

Inkjet printing

Antioxidants

Erythrocyte concentrates

\begin{abstract}
A B S T R A C T
Herein, we present the large scale fabrication of carbon nanotubes (CNT) electrodes supported on flexible polymeric sheets by subsequent multilayer inkjet printing of a silver layer for electrical connection, CNT layers as active electrode material and an insulation layer to define a stand-alone CNT active electrode area with high accuracy. Optical and electrochemical characterization using several redox mediators demonstrates the reproducibility of the electrode surfaces and their functionality even with a single inkjet printed CNT layer. These electrodes are targeted to the clinical sector for the determination of the antioxidant power (AOP) of biologically relevant fluids by pseudo-titration voltammetry. As a proof-ofconcept, the AOP of ascorbic acid solutions and biological samples such as erythrocyte concentrates (ECs) from different blood donors were determined demonstrating the potential use of the presented CNT sensors on ECs for blood transfusion purposes and the clinical sector.
\end{abstract}

(c) 2014 Elsevier B.V. All rights reserved.

\section{Introduction}

There is a great interest in measuring the antioxidant $(\mathrm{AO})$ defense system directly or indirectly in a fast, non-expensive and reliable way, not only in the human health sector (i.e. in blood and saliva), but also in the pharmaceutical and food industries. The reason for such interest is the fact that the antioxidant defense system, which is composed of enzymes, metal chelators, drugs and dietary AOs, normally counterbalances oxidative stress conditions by transforming excess of reactive oxygen species (ROS) into harmless compounds and complexes. Otherwise, an enhanced ROS level or a reduced activity of the AO defense system can lead to the damage of intracellular proteins, lipids, DNA, cellular membranes, cells and the development of several pathological conditions such as Parkinson, Alzheimer and cardiovascular diseases, neurological disorders, diabetes and cancer [1-3]. Therefore, monitoring the AO content in blood and saliva can provide relevant information about the status of the AO system and the overall health condition of a specific person. The latter implicates the measurement of a global AO content, denoted as total antioxidant capacity (TAC), rather than of single compounds. It is usually determined by several assays including the trolox equivalent antioxidant capacity

\footnotetext{
* Corresponding author. Tel.: +41 (0)21 69331 51; fax: +41 (0)21 6933667 .

E-mail address: hubert.girault@epfl.ch (H.H. Girault).
}

(TEAC) [4,5], the ferric reducing antioxidant power (FRAP) [6], the 2,2-diphenyl-1-picrylhydrazyl (DPPH) radical scavenging [7] and the oxygen radical absorbance capacity (ORAC) [8]. These approaches are based on the monitoring of colored or fluorescent compounds that are generated or degraded in the presence of AOs by means of electron transfer (ET) or hydrogen atom transfer (HAT) reactions. Comparing the obtained values with respect to a sample with a known concentration of a model AO, e.g. Trolox, the TAC can be expressed in several matrixes in terms of a common unit $[4-8]$.

Electrochemical assays that record signals of redox active species including AOs represent an alternative approach, which has been summarized in some recent reviews [9-11]. Cyclic voltammetry (CV) or linear sweep voltammetry (LSV) [12-14], differential pulse voltammetry $[15,16]$, square wave voltammetry $[17,18]$, the combination with HPLC detection $[19,20]$ and flow systems such as flow injection analysis (FIA) [9] have been used over a broad range of biological fluids. More recently, other assays such as the reducing antioxidant capacity evaluated by electrolysis (RACE) [21], the rapid electrochemical screening of antioxidant capacity (RESAC) [22] or microfluidic devices [23] were proposed. In addition, a new pseudo-titration voltammetry (PTV) concept where AOs that are rapidly oxidized at low oxidation potentials are mathematically separated from slow reacting and less relevant compounds has also been described [24]. 
In terms of electrode materials, carbon-based electrodes like carbon paste, glassy carbon or modified carbon composites are mainly used for electrochemical detection of AOs due to their well-known affinity for organic molecules, biocompatibility and low cost. Recently, carbon nanotubes (CNT) materials have attracted a lot of attention due to their high surface area, mechanical stability, electrical conductivity and electrochemical reactivity [25-27]. Indeed, it is expected that such type of materials will overcome drawbacks encountered in graphite electrodes such as inhomogeneous electrode surfaces, electrode fouling and high overpotentials for some electrode processes [27]. However, the properties and electrochemical characteristics of CNTs are still somewhat controversial [28-30]. A CNT is composed of $\mathrm{sp}^{2}$-hybridised, tubular carbon networks and can be of single wall (SWNT), double wall (DWNT) or multi wall (MWNT) type. Differences in their electrochemical behavior normally occur due to the nature of the CNTs, present functional groups, employed synthesis strategy, cleaning procedures and remaining metallic and carbonaceous impurities, storage conditions and aging [26,31-35].

Both graphite and CNT electrodes have been prepared by using screen-printed electrodes, thus allowing for batch fabrication of a multitude of disposable sensors [36]. A high resolution in the micrometer range is not easy to achieve by this technology and therefore a more precise technique is required to obtain highly reproducible sensors. Nowadays, inkjet printing of functional materials has become one very promising mask-free microfabrication technique that allows for a precise deposition of different materials such as CNTs [37-41]. Most of the functional CNT sensors have been deposited so far over a conductive metallic or carbonbased layer to ensure sufficient electrical conductivity among all the deposited CNTs. In addition, multiple printed layers ( $>10-30$ printed layers) are employed with the same aim. For instance, very recently, a Nafion/MWNT inkjet printed electrode (i.e. 15 inkjet printed layers) deposited on an indium-tin-oxide (ITO) coated polyethylene terephthalate (PET) substrate was employed for the detection of dopamine in blood with a high sensitivity $(0.1 \mu \mathrm{M})$ and a mean error less than $10 \%$ for 5 tested electrodes [39]. To date, stand-alone inkjet printed CNT electrodes with proper electrochemical behavior and fully functional as amperometric sensors after a single printed layer are still rare.

In this study, we use a commercially available DWNTs dispersion suitable for inkjet printing (IJP) for the fabrication of standalone CNT electrodes with high electrical conductivity and electrochemical activity thanks to the percolation of the randomly spread and flat CNT networks as corroborated by scanning electron microscopy (SEM) analysis. A silver layer for electrical connections and an insulating layer to precisely define the active electrode area complete the CNT sensor. Optical microscopic, electrical and electrochemical analysis demonstrated a high reproducibility of the fabrication process of more than 350 units per batch. The developed amperometric sensors were applied in combination with the PTV method for measuring the AOP of ascorbic acid solutions (i.e. a model AO) and in real samples such as erythrocyte concentrates (ECs), a labile blood product widely used in transfusion medicine, donated by two healthy persons and targeted for blood donation. The latter is of high relevance in blood product research and management since erythrocytes suffer from storage lesions, during storage at $4{ }^{\circ} \mathrm{C}$, which alter lipids, proteins, metabolism, and biomechanical properties of the cells [42-48], resulting in potential adverse effects upon transfusion [47-52]. Erythrocytes are enucleated cells not able to synthesize proteins, which requires a solid system (like the repair or destroy box) $[53,54]$ to protect the cells against oxidative stress. Thus in order to take the proper actions to diminish such effects, the measurement of AOs in ECs has to be performed in a reliable, cost-effective and precise way.

\section{Experimental section}

\subsection{Chemicals}

Ferrocenemethanol (FcMeOH; Sigma-Aldrich, Buchs, Switzerland), hexaammine ruthenium chloride $\left(\left[\mathrm{Ru}\left(\mathrm{NH}_{3}\right)_{6}\right] \mathrm{Cl}_{3} ;\right.$ Acros, Renningen, Germany), potassium hexachloroiridate(III) $\left(\mathrm{K}_{3} \mathrm{IrCl}_{6}\right.$; Alfa Aesar, Karlsruhe, Germany), potassium chloride (KCl; SigmaAldrich, Buchs, Switzerland) and L(+)-Ascorbic acid (AA; Riedelde Haën) were used as received and were of analytical grade. Deionized water was produced by a Milli-Q plus 185 model (Millipore, Zug, Switzerland). Jettable nano silver EMD5603 (w/w 20\%), jettable insulator EMD6201 (both Sun Chemical, Carlstadt, USA) and the CNT dispersion BSI.B12212 (Brewer Science, Rolla, USA) were used as inks for IJP. As specified by the manufacturer, the CNTs are mostly DWNTs with an unknown amount of SWNTs with lengths $L=763.08 \pm 595.16 \mathrm{~nm}$, diameters $d=1.56 \pm 0.56 \mathrm{~nm}$ and an aspect ratio $L / d=322.6 \pm 454.5$. The CNTs were functionalized by the manufacturer with 1-pyrenemethylamine-3,6,8-trisulfonic acid groups to ensure a stable dispersion free of agglomerates.

\subsection{Preparation of CNT sensors}

CNT sensors were prepared on Kapton $\mathrm{HN}^{\oplus}$ foils (polyimide (PI); $125 \mu \mathrm{m}$-thick; Goodfellow, Huntingdon, England) by a multilayer inkjet printing process (vide infra) and by using the drop-on-demand DMP-2831 materials printer (Dimatix Fujifilm, Santa Clara, CA, USA). This printer allows simultaneous printing with up to 16 nozzles and providing droplet volumes of 1 and $10 \mathrm{pL}$. During IJP of both conductive inks, i.e. Ag and CNT, the substrate temperature was increased for a faster evaporation of the ink carrier solvents and therefore reaching an increase in the pattern resolution. The Ag and CNT patterns were cured for $30 \mathrm{~min}$ at $200{ }^{\circ} \mathrm{C}$ and $120^{\circ} \mathrm{C}$, respectively, with a heating and cooling rate of $1-$ $2{ }^{\circ} \mathrm{C} \mathrm{min}^{-1}$. The insulating UV curable ink was printed under simultaneous UV exposition. The latter was achieved by a custom-made modification of the DMP-2831 system by mounting a light guide from an Omnicure ${ }^{\circledR}$ S2000 mercury UV lamp (Lumen Dynamics, Mississauga, Ontario, Canada) into the DMP-2831 print head. The Dimatix software allows opening of the UV light guide shutter during printing in order to polymerize the UV curable ink just after it has been deposited on the substrate. Printing parameters such as jetting frequency, waveform, voltage, cartridge temperature, and cleaning cycles were adjusted for optimum printing performance of each ink. The dimensions of the patterns were investigated by laser scanning microscopy (LSM) in reflection mode using a Keyence VK 8700 (Keyence, Osaka, Japan) and by SEM using a LEO 1550 (Carl Zeiss, Jena, Germany).

\subsection{Preparation of erythrocyte concentrate}

ECs were prepared from whole blood donations. Briefly, $450 \pm 50 \mathrm{~mL}$ of blood from two healthy volunteer donors were mixed with $63 \mathrm{~mL}$ of citrate phosphate dextrose anticoagulant solution and left at $22{ }^{\circ} \mathrm{C}$ overnight. All blood components (i.e. erythrocytes, plasma and white blood cell- and platelet-containing buffy coat) were separated upon centrifugation at $3500 \mathrm{~g}$ for $14 \mathrm{~min}$. The separated components were then distributed among the sterile inter-connected blood bags by applying a semi-automated pressure on the centrifuged original blood donation bag. Erythrocytes were then transferred into a SAG- $\mathrm{M}^{\mathrm{TM}}$-containing bag to a total volume of $275 \pm 75 \mathrm{~mL}$ and a hematocrit level of $0.6 \pm 0.1 v / v$. A leukodepletion step was performed by filtration. ECs that did not meet the quality criteria for blood transfusion, i.e. a low hemoglobin content or a too small volume, were used un- 
der the signed assent of blood donors. Each $100 \mathrm{~mL}$ of the additive solution SAG-M ${ }^{\mathrm{TM}}$ contains $0.9 \mathrm{~g}$ of dextrose, $0.877 \mathrm{~g}$ of $\mathrm{NaCl}$, $0.0169 \mathrm{~g}$ of adenine and $0.525 \mathrm{~g}$ of Mannitol.

\subsection{Electrochemical measurements}

CV and LSV were carried out with Autolab potentiostats PGSTAT101 and PGSTAT128N (Metrohm, Eindhoven, The Netherlands) in a three electrode arrangement using the CNT sensors as working electrodes, a Pt wire as counter electrode and either a $\mathrm{Ag} / \mathrm{AgCl} / 1 \mathrm{M} \mathrm{KCl}$ electrode as reference electrode (RE) or a $\mathrm{Ag}$ wire as quasi $\mathrm{RE}(\mathrm{QRE})$. A scan rate $v$ of $100 \mathrm{mV} \mathrm{s}^{-1}$ was employed in all experiments unless stated otherwise. No pretreatment of the CNT electrodes was performed. Storage in air over several weeks did not change the electrochemical response of the CNT electrodes. All measurements were performed under ambient conditions at room temperature.

\section{Results and discussion}

\subsection{Design and multilayer inkjet-printing for the large-scale production of CNT sensors}

For the highly reproducible, large-scale production of amperometric CNT sensors, a three layer IJP process was developed by using a drop-on-demand inkjet printer (Fig. 1). Firstly, a conductive Ag pattern consisting of a long band and a rectangle was prepared to establish the electrical connection of the sensor with the potentiostat. Four crosses at the edges of the pattern were placed for

(a)

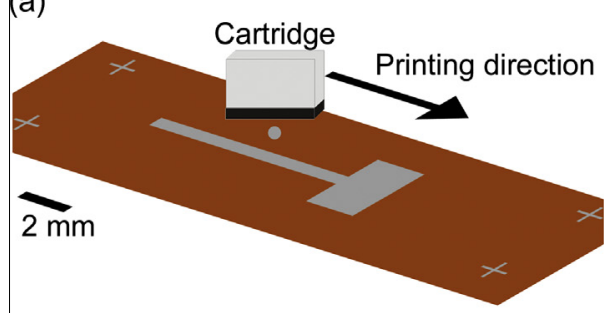

(b)
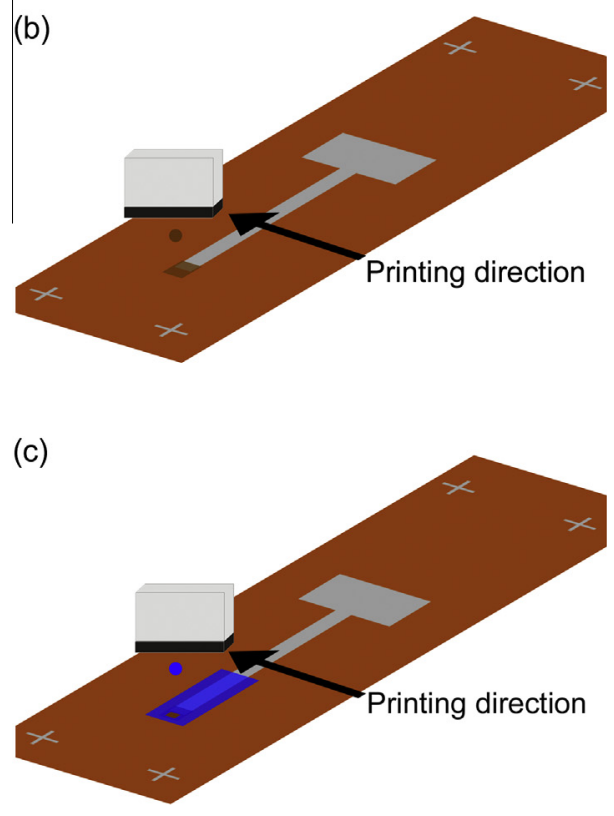

Fig. 1. Schematic representation of the large-scale production of CNT electrodes by multilayer inkjet printing. (a) Ag layer for electrical conduction; (b) CNT layer as active electrode material; (c) insulation layer to define the active electrode area. subsequent alignment procedures in the printer setup, for instance when replacing the substrate after curing in an oven, when exchanging the cartridge or adjusting of the printing resolution, which is in case of the used printer the change of the angle of the linear 16 nozzles array. Furthermore, the crosses are used to cut the individual sensors into individual strips prior to use. After curing the Ag inkjet printed pattern, a CNT structure of $1.7 \mathrm{~mm} \times 1.5 \mathrm{~mm}$ was deposited in such way that a CNT area of approximately $1.7 \mathrm{~mm} \times 0.65 \mathrm{~mm}$ overlapped the end of the Ag band assuring the electrical connection. The printing direction was rotated by $90^{\circ}$ with respect to the previous layer. After curing of the inkjet printed CNT pattern, a UV curable ink was employed to create an insulating layer covering partially both the Ag and the CNT films and leaving a theoretical rectangular stand-alone CNT area of $0.3575 \mathrm{~mm}^{2}$ uncovered as active electrode surface. For electrochemical experiments individual sensors were dipped vertically in the electrolyte solution in order to ensure a complete wetting of the exposed CNT surface. The Ag layer was protected thanks to the insulation layer.

\subsection{Optical characterization and surface resistance measurement of the inkjet printed CNT sensors}

The final CNT sensors obtained after the multilayer IJP process are shown in Fig. 2. The optical micrograph in Fig. 2a shows clearly the Ag pattern, the insulation layer as well as the active CNT electrode area. Batch production can easily be performed by up-scaling the number of desired patterns (Fig. 2b). The sample holder, socalled platen, allows the placement of a substrate of $203.2 \mathrm{~mm}$ (8 in.) $\times 279.4 \mathrm{~mm}(11 \mathrm{in}$.) size, which means that up to 368 sensors (pitch size $6 \mathrm{~mm} \times 24 \mathrm{~mm}$ ) can be printed in three steps. However, the pitch dimensions could be even smaller (Fig. 2b). The high resolution of the printing process can be seen by the laser scanning micrographs of the active CNT electrode area in Fig. 2c. The $\mathrm{Ag}$ pattern can be identified in the bottom of the image whereas the insulation layer is clearly identifiable by the printed bands. These bands are generated by using simultaneously three nozzles during printing that release droplets in close proximity to each other (drop spacing $50 \mu \mathrm{m}$ ) and forming one merged printed line. Slightly delayed after the deposition of droplets, the UV photopolymerization of the specific band takes place when the UV light guide passes over this area. Since the CNTs form a transparent layer, it cannot easily be identified by laser scanning microscopy (LSM) but by SEM (see Fig. $2 \mathrm{~d}$ and Supporting Information SI-1). The measured geometric area $A_{\mathrm{g}}$ of the electrode is $0.358 \mathrm{~mm}^{2}$, which is very close to the theoretical one defined by the printing parameters (vide supra). CNT electrodes were prepared with 1-, 2- and 4-inkjet-printed layers (IJPL). Please note that 1-IJPL does not correspond to a monolayer of CNTs, but to a thin felt layer. Fig. 2d shows a SEM of a 4-IJPL CNT pattern on Ag. A homogenous surface coverage of flat and randomly oriented CNTs can easily be identified. The measured sheet resistant by using a four-point probe system of a 4 -IJPL CNT electrode is $707.9 \pm 17.9 \Omega \mathrm{sq}^{-1}$ which is in good agreement to the value of $550 \Omega \mathrm{sq}^{-1}$ (85\% transparency at $550 \mathrm{~nm}$ ) reported by the manufacturer for an equal number of IJPL. Surface resistance measurements with a lower number of IJPL patterns were unsuccessful mainly due to lack of electrical connection between the four-point probes and the less populated or thinner films. Indeed, the latter might suggest that a minimum of 4-IJPL is required for having a proper electrical conductivity via percolation, but as demonstrated by cyclic voltammetry experiments (vide infra) this is not the case.

\subsection{Voltammetric characterization of the inkjet printed CNT electrodes}

CVs were performed in $2 \mathrm{mM} F \mathrm{FMeOH}$ and $0.1 \mathrm{M} \mathrm{KCl}$ at a scan rate of $v=100 \mathrm{mV} \mathrm{s}^{-1}$ using various 1-, 2- and 4-IJPL CNT 

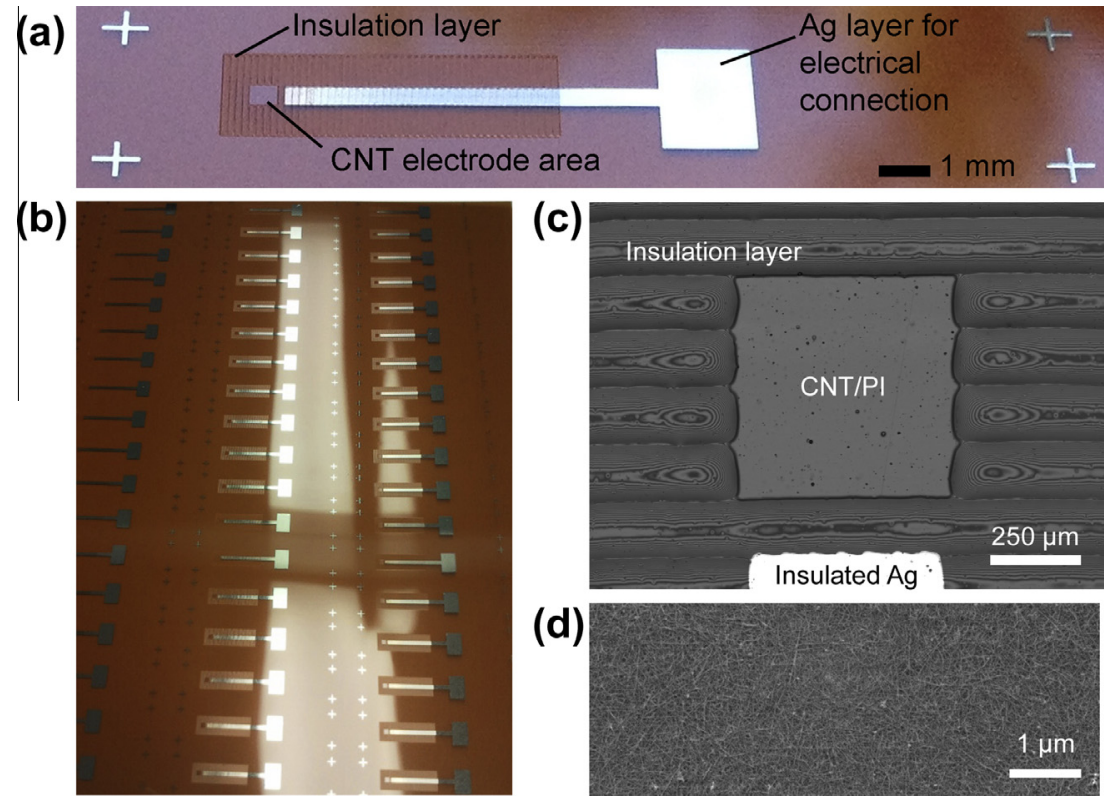

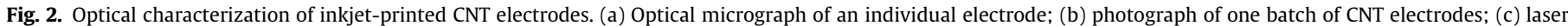
scanning micrograph of active electrode area; (d) scanning electron micrograph of the inkjet-printed CNT structure.

electrodes without any pretreatment of the electrode surface (Fig. 3a-c). A peak waved shape, typical for electrochemical processes limited by semi-infinite linear diffusion was recorded in all cases for the oxidation of $\mathrm{FcMeOH}$ and the reduction of generated $\mathrm{FcMeOH}^{+}$with a formal potential $\hat{E}^{0}=0.193 \mathrm{~V}$ vs.
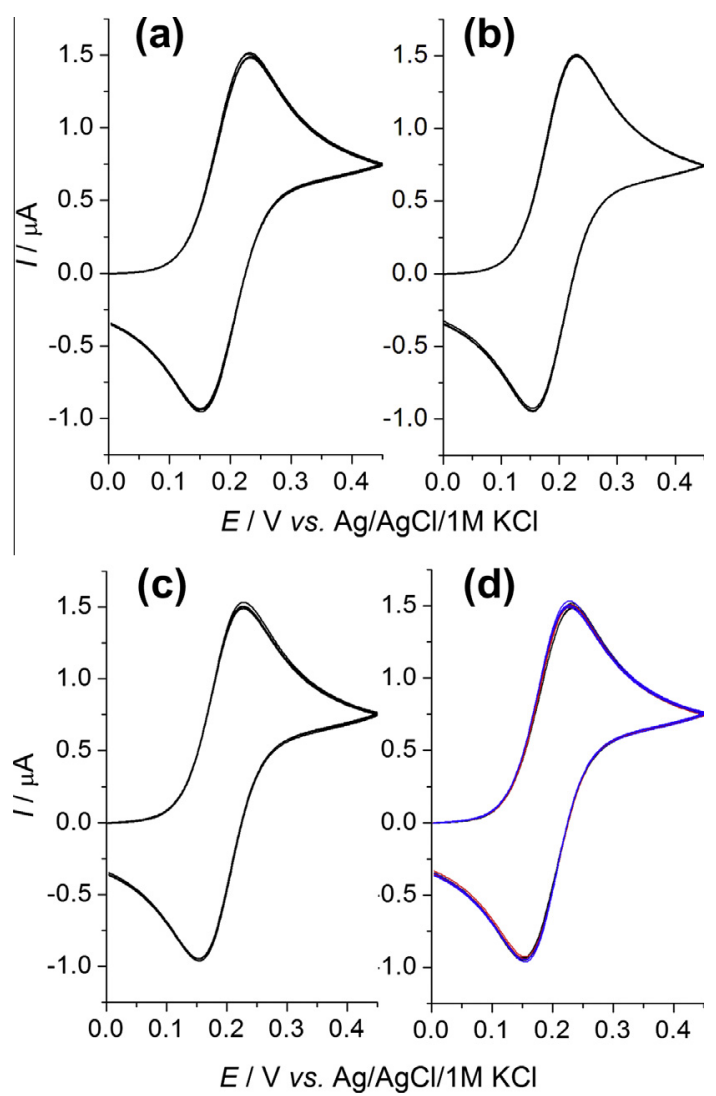

Fig. 3. Influence of the number of IJPL of CNT on the CVs (first cycles) in $2 \mathrm{mM}$ FCMeOH and $0.1 \mathrm{M} \mathrm{KCl}$ using overlayed four 1-IJPL CNT sensors (a), overlayed six 2IJPL CNT sensors (b) and overlayed four 4-IJPL CNT sensors (c). (d) Overlayed plot of (a)-(c). Scan rate $v=100 \mathrm{mV} \mathrm{s}^{-1}$.
$\mathrm{Ag} / \mathrm{AgCl} / 1 \mathrm{M} \mathrm{KCl}$. The almost identical electrode response obtained for all tested electrodes (see Fig. 3d) demonstrates a reproducible electrode surface preparation achieved among the different CNT electrodes of identical IJPL. When comparing the response among the CNT electrodes with different number of IJPL, at $100 \mathrm{mV} \mathrm{s}^{-1}$ all the results look almost identical (Fig. 3d), however as shown in Fig. $4 \mathrm{a}$ and SI-2 some differences are observed when using higher scan rates.

In order to characterise the electrochemical response of the different electrodes, CVs at different scan rates from 10 to $1000 \mathrm{mV} \mathrm{s}^{-1}$ were performed. Fig. 4a shows the results for one 2IJPL CNT electrode while the plots for 1- and 4-IJPL CNT probes can be found in SI-2. The anodic peak currents $I_{\mathrm{pa}}$ are a linear function of the square root of the scan rate $\left(v^{1 / 2}\right)$ for all printed CNT layers (SI-2), corroborating that the electrochemical process is controlled by semi-infinite linear diffusion.

As can be seen from Table 1, this observed voltammetric behavior is not completely described by the Randles-Sevcik equation for electrochemically reversible systems, since the slopes obtained

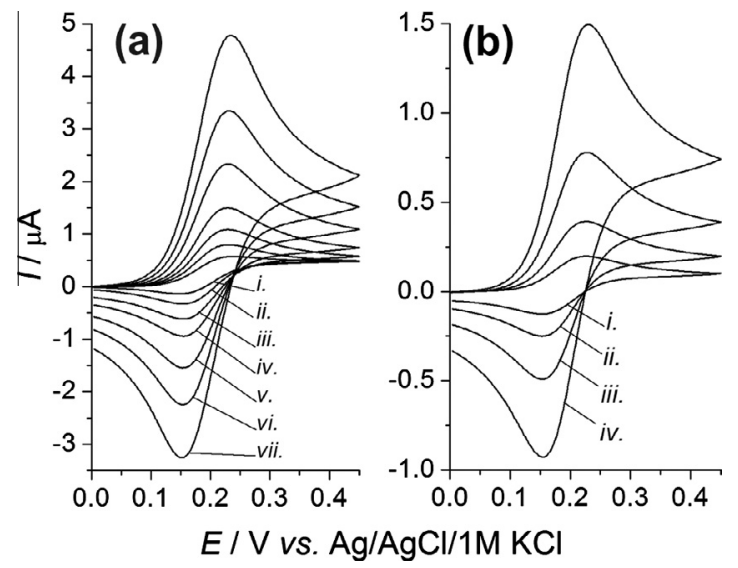

Fig. 4. (a) Scan rate and (b) concentration dependence of the CVs (first cycle) in $2 \mathrm{mM}$ FCMeOH using one 2-IJPL CNT sensor at various $v$ in $\mathrm{mV} \mathrm{s}^{-1}: 10(i), 25(i i), 50$ (iii), $100\left(\right.$ iv.), $250(v), 500$ (vi), 1000 (vii) and various $c_{\mathrm{FcMeOH}}$ in mM: 0.25 (i), 0.5 (ii), 1 (iii), 2 (iv). Supporting electrolyte $0.1 \mathrm{M} \mathrm{KCl}$. Other experimental conditions for (a) $2 \mathrm{mM} \mathrm{FCMeOH}$ and for (b) $v=100 \mathrm{mV} \mathrm{s}^{-1}$. 
experimentally for each plot $I_{\mathrm{pa}} v s . v^{1 / 2}$ differ from the theoretical one expressed as a function of the Faraday constant $(F)$, ideal gas constant $(R)$, temperature $(T)$, the number of transferred electrons $(n)$, the diffusion coefficient $(D)$, the concentration $(c)$, the microscopic area of the electrode $\left(A_{\mathrm{m}}\right)$ and a constant equal to 0.4463 (see SI-3). Therefore, by employing the slope obtained experimentally for each $I_{\mathrm{pa}}$ and $v^{1 / 2}$ data set, the difference between the expected constant value and the experimental one was determined (i.e. by assuming $A_{\mathrm{g}}=A_{\mathrm{m}}=3.58 \times 10^{-3} \mathrm{~cm}^{2}, \quad D(\mathrm{FcMeOH})=$ $7.8 \times 10^{-6} \mathrm{~cm}^{2} \mathrm{~s}^{-1}$ [55], $T=298 \mathrm{~K}, c=2 \mathrm{mM}$, see Table 1 ). As it can be seen in Table 1, in all cases a smaller value than the theoretical one (i.e. 0.4463) was found, and it is more pronounced for CNT electrodes composed by 1 -IJPL. In addition, the peak potential separation $\Delta E_{\mathrm{p}}$ between the anodic $\left(E_{\mathrm{pa}}\right)$ and cathodic $\left(E_{\mathrm{pc}}\right)$ peaks at various $v$ exceed the theoretical value of $59 \mathrm{mV}$ for a fast one-electron transfer reaction by about $26.6 \mathrm{mV}, 16.5 \mathrm{mV}$ and $15 \mathrm{mV}$ for 1-, 2- and 4-IJPL, respectively (see Table 1 ). All these results can be explained by the $I R$ drop along the electrode itself, the fact that the electrodes present microscopic inhomogeneities that lead to non-linear diffusion effects resulting in a peak to peak separation widening and also to some kinetic limitations [56]. Indeed, such inhomogeneities might also depend on the generated porous CNT network, the specific local reactivity of the DWNTs and the influence of functional groups on the electrochemical response of the IJP CNT electrodes. For instance, the increase on the number of IJPL improves the homogeneity of the electrode and the number of active sites. Still, even with a single IJPL CNT electrode, a functional stand-alone CNT amperometric sensor with an acceptable electrochemical response is obtained, which to the best of our knowledge is exceptional (vide infra). Furthermore, the ratio between $I_{\mathrm{pa}}$ and the cathodic peak current $I_{\mathrm{pc}}$ is around 1 for all cases, and $I_{\mathrm{pa}}$ is proportional to the concentration of $\mathrm{FcMeOH}$ as shown in Fig. 4b. The Randles-Sevcik constant based on the concentration dependence of FcMeOH (Fig. 4b) is equal to 0.389, which is in good agreement with the value of Table 1 .

Additionally, the influence of the redox mediator charge on the response of the redox mediator was investigated. Indeed, highly reproducible CVs with the IJP CNT amperometric sensors for the oxidation of $\mathrm{IrCl}_{6}^{3-}$ as a negatively charged (Fig. 5a) and the reduction of $\left[\mathrm{Ru}\left(\mathrm{NH}_{3}\right)_{6}\right]^{3+}$ as a positively charged (Fig. $5 \mathrm{~b}$ ) redox species for several 2-IJPL CNT electrodes were obtained. It can be seen that an increase on the peak to peak separation is obtained for the negatively charged $\mathrm{IrCl}_{6}^{3-}$ in comparison to $\mathrm{FcMeOH}$ and $\left[\mathrm{Ru}\left(\mathrm{NH}_{3}\right)_{6}\right]^{3+}$. When calculating the constant term from the Randles-Sevcik equation, a value equal to 0.420 was obtained for the $\left[\mathrm{Ru}\left(\mathrm{NH}_{3}\right)_{6}\right]^{3+}$ $\left(D\left(\left[\mathrm{Ru}\left(\mathrm{NH}_{3}\right)_{6}\right]^{3+}\right)=5.3 \times 10^{-6} \mathrm{~cm}^{2} \mathrm{~s}^{-1}[55]\right.$, and a value of 0.367 for $\left.\mathrm{IrCl}_{6}^{3-}, D\left(\mathrm{IrCl}_{6}^{3-}\right)=7.5 \times 10^{-6} \mathrm{~cm}^{2} \mathrm{~s}^{-1}[57]\right)$. These data might suggest a favored electron transfer process between the negatively charged CNT (vide supra) and the positively charged $\left[\mathrm{Ru}\left(\mathrm{NH}_{3}\right)_{6}\right]^{3+}$ species and a slower process for the negatively charged $\mathrm{IrCl}_{6}^{3-}$.

\subsection{Antioxidant measurements}

AA is an important dietary AO for the human organism and present in many biological systems as well as in industrial

Table 1

Parameters of CVs from Fig. 4a and SI-2.

\begin{tabular}{lllll}
\hline IJPL & Randles-Sevcik const. & $b \times 10^{6} / \mathrm{A}$ & $\Delta E_{\mathrm{p}} / \mathrm{mV}$ & $I_{\mathrm{pa}} / I_{\mathrm{pc}}$ \\
\hline 1 & $0.327 \pm 0.003$ & $0.165 \pm 0.055$ & $85.6 \pm 11.1$ & $1.052 \pm 0.018$ \\
2 & $0.367 \pm 0.002$ & $0.061 \pm 0.034$ & $75.7 \pm 6$ & $1.055 \pm 0.021$ \\
4 & $0.352 \pm 0.018$ & $0.085 \pm 0.018$ & $74 \pm 5.7$ & $0.996 \pm 0.020$
\end{tabular}

a Randles-Sevcik const. $=m /\left(n^{3 / 2} \times A_{\mathrm{g}} \times D(\mathrm{FcMeOH})^{1 / 2} \times c_{\mathrm{FcMeOH}} \times R T^{-1 / 2} \times F^{3 / 2}\right)$, and $m$ was obtained from the curves $I_{\mathrm{pa}}=m v^{1 / 2}+b$ for each IJPL CNT electrode (SI-2 and SI-3).

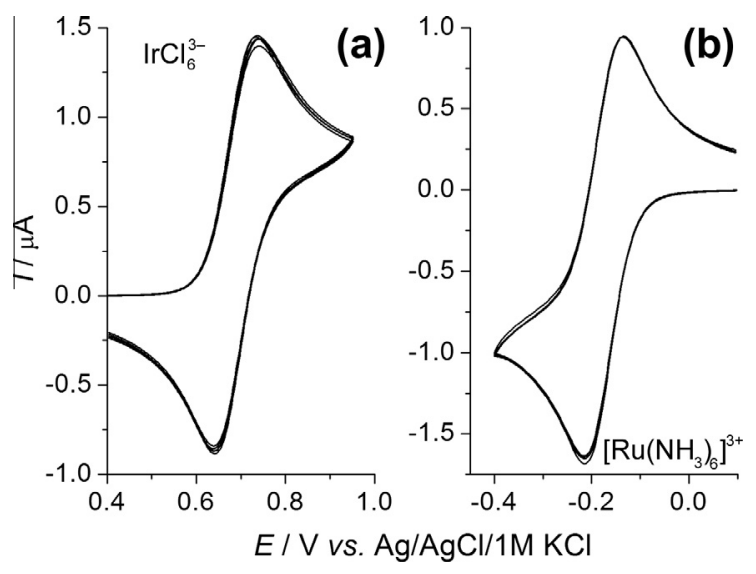

Fig. 5. Redox mediator charge dependence of the $\mathrm{CVs}$ (first cycle) in (a) $2 \mathrm{mM} \mathrm{IrCl}_{6}^{3-}$ (five 2-IJPL CNT sensors) and (b) $2 \mathrm{mM}\left[\mathrm{Ru}\left(\mathrm{NH}_{3}\right)_{6}\right]^{3+}$ (four 2-IJPL CNT sensors, degassed solution). Supporting electrolyte $0.1 \mathrm{M} \mathrm{KCl} . v=100 \mathrm{mV} \mathrm{s}^{-1} . \Delta E_{\mathrm{p}}=\left(E_{\mathrm{pa}}\right.$ $\left.-E_{\mathrm{pc}}\right)=95.7 \pm 3.4 \mathrm{mV}$ and $74.4 \pm 2.5 \mathrm{mV}$, respectively.

products. Since it can be detected electrochemically at carbon based electrodes, it was used herein as a model system for AO detection. Two AA concentrations, namely $0.5 \mathrm{mM}$ and $1.0 \mathrm{mM}$, were investigated under physiological conditions using 1-IJPL CNT sensors. LSVs were carried out from $0.0 \mathrm{~V}$ to $1.3 \mathrm{~V}$ vs. a $\mathrm{Ag} /$ $\mathrm{AgCl} / 1 \mathrm{M} \mathrm{KCl}$ reference electrode and the results are shown in Fig. 6. A clear oxidation peak of AA can be obtained at $E_{\mathrm{pa}}=0.508 \pm 0.005 \mathrm{~V}$ for both concentrations. The values for $I_{\mathrm{pa}}$ vary only by $2 \%$ (a) and $1 \%$ (b), respectively, demonstrating that the inkjet-printed CNT sensors can be applied as reliable tool to detect AA in aqueous phosphate buffered systems. For each measurement, a fresh CNT strip is used to avoid any influence from possible electrode fouling. Moreover, the presented inkjet printing batch fabrication process affords for disposable or single use CNT sensors. Recently, a pseudo-titration method was introduced to separate the most relevant AOs, which react quickly at low oxidation potentials, from less reactive species in solution [24]. A mathematical treatment of the LSVs is performed by multiplying the measured currents with a Fermi-Dirac function $f(E)$ in order to measure a modulated current $I_{\text {mod: }}$ :

$I_{\text {mod }}=I \cdot f(E)=I \cdot \frac{1}{1+\exp \left(\frac{\lambda n F\left(E-E_{\text {threshold }}\right)}{R T}\right)}$

where $E$ is the applied potential. Two adjustable parameters, namely $\lambda$ and $E_{\text {threshold, }}$ are used as a factor modulating the shape of the LSV and as a potential offset to shift the curve towards smaller or higher potentials, respectively (SI-4) [24]. An antioxidant power (AOP) can be calculated by integrating $I_{\text {mod }}$ and is presented in $\mathrm{nW}$ (Eq. (2)).

$\mathrm{AOP}=\int I_{\mathrm{mod}} d E$

For $0.5 \mathrm{mM}$ AA an AOP of $128 \pm 3 \mathrm{nW}$ is calculated whereas $253 \pm 5 \mathrm{nW}$ is obtained for $1 \mathrm{mM}$ AA that describes a factor of 1.97 as expected from the concentration ratio (i.e. 2). Please note, that these results depend on the selected values for $\lambda$ and $E_{\text {threshold }}$ and also on the electrode area since the measured current is proportional to the latter parameter. Therefore, measurements of different systems can only be compared under equal conditions.

The PTV concept was applied to two EC samples of two donors (so-called samples 1 and 2) using 1- and 4-IJPL CNT sensors (Fig. 7). The samples were prepared under the same conditions and hence variations in the electrochemical response refer to the ECs' compositions or age. For both CNT sensor types two broad oxidation 

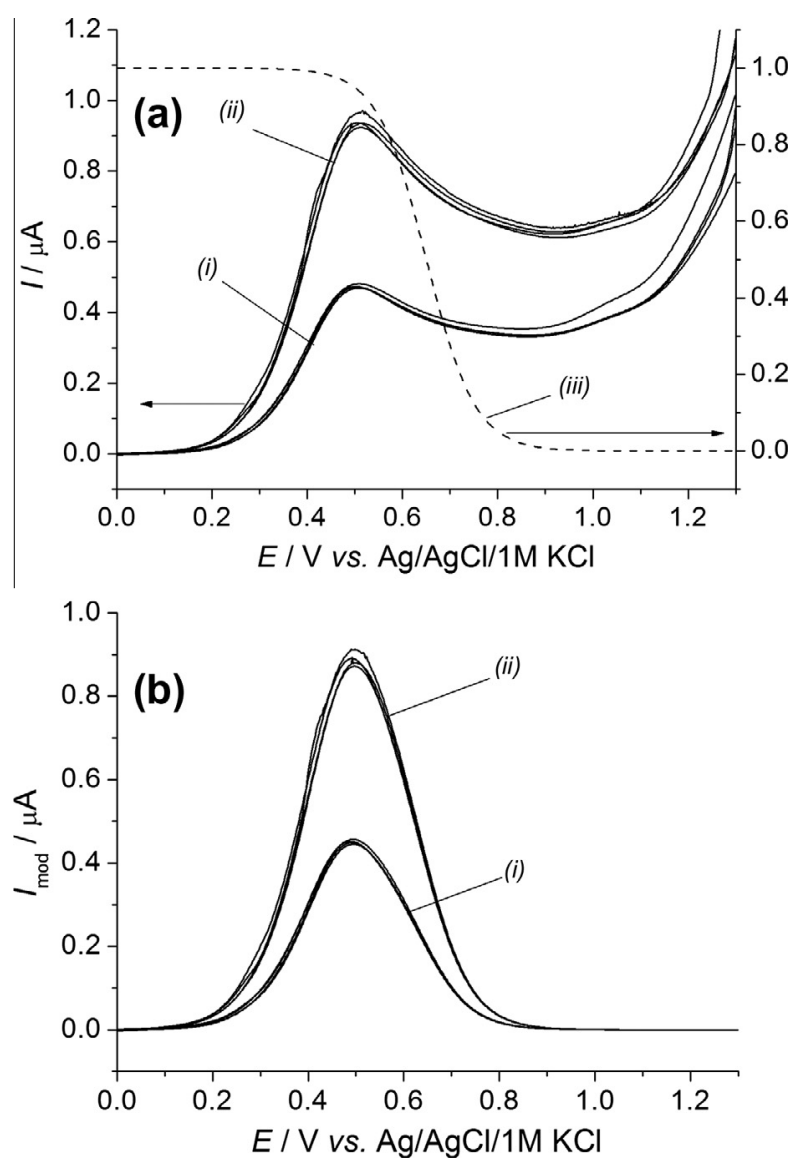

Fig. 6. (a) LSVs in $0.5 \mathrm{mM} \mathrm{AA}$ ( $i$ ) and in $1 \mathrm{mM}$ AA (ii) using for each concentration four 1-IJPL CNT sensors. Fermi-Dirac plot $f(E)$ (dashed line, iii) using $E_{\text {thresh- }}$ old $=0.65 \mathrm{~V}$ and $\lambda=0.5$ which is used for the pseudo-titration. b) Obtained pseudotitration curves from the LSVs shown in Fig. 6a. Supporting electrolyte $50 \mathrm{mM}$ phosphate buffer, $v=100 \mathrm{mV} \mathrm{s}^{-1}$.

waves can be identified in both samples, due to the presence of antioxidant species such as uric acid, glutathione and others. Further studies will be performed to determine the identity of the detected antioxidant species. The measured background signal of a pure SAG-M ${ }^{\mathrm{TM}}$ solution can clearly be separated from the LSVs in the EC samples demonstrating that the measured signals are due only to the ECs' nature. The LSVs of samples 1 and 2 can clearly be distinguished by the measured currents most likely due to different AO contents. However, although both used CNT sensors gave the same trend, 1-IJPL CNT electrodes suffer a large variation among each other and some signals measured in both samples even overlap (Fig. 7a). In contrast, 4-IJPL CNT electrodes show a much better reproducibility (Fig. 7b). Although both probe types show very similar electrochemical properties with standard redox mediators, a clear discrimination can be seen when measuring AOs or electroactive species with slower or more complex kinetics.

Still, by comparing the LSVs measured with 1-IJPL, 4-IJPL with a commercial standard screen printed carbon electrode, it is clear that the CNT sensors show apparently faster kinetics and a catalytic effect compared to the carbon paste surface as can be seen in Fig. 7c by the more pronounced slopes and lower oxidation potentials, respectively. For a better comparison, all current values were normalized by the geometric area of each electrode. These results show clearly the advantages of IJP CNT sensors over screenprinted carbon paste electrodes when testing blood samples.

The AOPs of the two EC samples were calculated by using the PTV method and are shown in Table 2. According to these results,
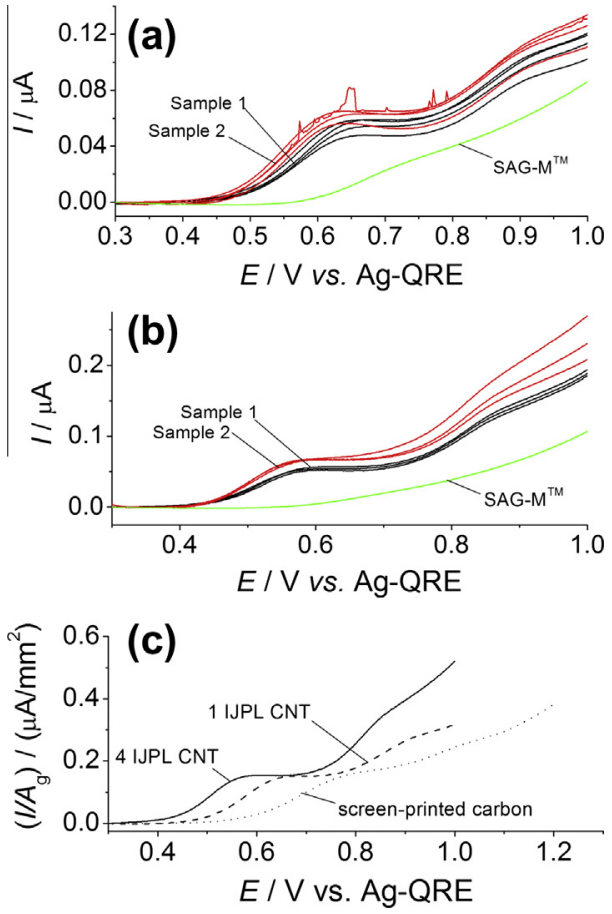

Fig. 7. LSVs in ECs of two different donors using four 1-IJPL CNT sensors (a) and three 4-IJPL CNT sensors (b) for each sample. (c) Normalized LSVs in sample 1 using one 1-IJPL, one 4-IJPL CNT electrode and a commercial screen printed carbon paste electrode. Scan rate $100 \mathrm{mV} \mathrm{s}^{-1}$.

Table 2

Antioxidant power of two erythrocyte concentrates measured with four 1-IJPL and three 4-IJPL CNT sensors. $E_{\text {threshold }}=0.65 \mathrm{~V}$ and $\lambda=0.5$.

\begin{tabular}{llr}
\hline IJPL & Sample & \multicolumn{1}{c}{ AOP $(\mathrm{nW})$} \\
\hline 1 & 1 & $5.74 \pm 0.65$ \\
1 & 2 & $7.30 \pm 0.81$ \\
4 & 1 & $8.60 \pm 0.45$ \\
4 & 2 & $10.89 \pm 0.01$ \\
\hline
\end{tabular}

which represent the average of four and three individual measurements using fresh 1-IJPL and 4-IJPL CNT sensors, respectively, sample 2 has an AOP 1.27 times higher than the one observed with sample 1 . When comparing the results obtained between the two different IJPL CNT sensors, a 50\% larger value is obtained for all samples with the 4-IJPL CNT sensors due to a shift of the oxidation potential to lower values. The latter is an important issue, since for slow or complex electron transfer processes, the conductivity of the stand-alone CNT electrodes play an important role, while it is almost negligible with standard redox mediators.

Finally, it has to be noted that the AOP varies from person to person, and is a dynamic value that can only be used as an indicator of health or blood sample status by performing long-term studies to establish normal or healthy range values. Indeed, ECs are usually stored for less than 42 days since aging of the red blood cells due to oxidative stress among other reasons, usually takes place. However, two weeks old EC units can already start to suffer from these aging effects related with higher risk for postoperative complications [50,52]. The PTV concept together with the IJP CNT sensors can be a very useful system to help understanding the appearance of storage lesions linked to oxidative stress and also to probe the actual AOP before, during and at the end of the storage period. In this way, an important contribution to improve the quality of medical red blood cell transfusions can be made. 


\section{Conclusion}

The batch production of stand-alone carbon nanotubes sensors by multilayer inkjet printing and their use as amperometric AO sensors was demonstrated. Since commercially available inks and a commercial drop-on-demand inkjet printer machine were used, the concept is very attractive for many analytical chemistry applications at academic and industrial levels. Optical microscopy, scanning electron microscopy and cyclic voltammetry of single and multiple inkjet printed layers of carbon nanotubes electrodes showed highly reproducible and homogeneous active electrode areas that present a quasi-reversible electrochemical behavior adequate for amperometric sensing. It is important to notice that the carbon nanotubes were deposited on insulating polyimide sheets and thus all obtained signals came only from the stand-alone carbon nanotubes structure itself. The pseudo titration voltammetric method was used to measure the antioxidant power in erythrocyte concentrates showing the capabilities and advantages of the present inkjet printed carbon nanotubes electrodes as amperometric sensors. These sensors are targeted to the clinical sector (i.e. blood samples) and therefore dedicated for single or disposable use to avoid any influence from possible electrode fouling. The latter becomes possible only by a precise, reproducible and large-scale batch fabrication technique such as inkjet printing.

\section{Acknowledgments}

The authors are very thankful to Adam Wood and James Lamb from Brewer Science for the provided information and technical support about the CNT ink. This work was supported by the Swiss Commission for Technology and Innovation (CTI, Project No. 13841.2PFLS-LS).

\section{Appendix A. Supplementary material}

Supplementary data include laser scanning and scanning electron microscopy photographs of the inkjet printed CNTs, as well as, more details about the characterization of the electrochemical response of the inkjet printed CNTs, and the PTV methodology. Supplementary data associated with this article can be found, in the online version, at http://dx.doi.org/10.1016/j.jelechem. 2013.12.027.

\section{References}

[1] B.N. Ames, M.K. Shigenaga, T.M. Hagen, Oxidants, antioxidants, and the degenerative diseases of aging, Proc. Natl. Acad. Sci. USA 90 (1993) 7915-7922.

[2] M. Valko, D. Leibfritz, J. Moncol, M.T.D. Cronin, M. Mazur, J. Telser, Free radicals and antioxidants in normal physiological functions and human disease, Int. J. Biochem. Cell Biol. 39 (2007) 44-84.

[3] B. Halliwell, J. Gutteridge, Free Radicals in Biology and Medicine, fourth ed., Oxford University Press, 2007.

[4] N.J. Miller, C. Rice-Evans, M.J. Davies, V. Gopinathan, A. Milner, A novel method for measuring antioxidant capacity and its application to monitoring the antioxidant status in premature neonates, Clin. Sci. 84 (1993) 407-412.

[5] R. Van Den Berg, G.R.M.M. Haenen, H. Van Den Berg, A. Bast, Applicability of an improved Trolox equivalent antioxidant capacity (TEAC) assay for evaluation of antioxidant capacity measurements of mixtures, Food Chem. 66 (1999) 511-517.

[6] I.F.F. Benzie, J.J. Strain, The ferric reducing ability of plasma (FRAP) as a measure of 'antioxidant power': the FRAP assay, Anal. Biochem. 239 (1996) $70-76$.

[7] O.P. Sharma, T.K. Bhat, DPPH antioxidant assay revisited, Food Chem. 113 (2009) 1202-1205.

[8] G. Cao, H.M. Alessio, R.G. Cutler, Oxygen-radical absorbance capacity assay for antioxidants, Free Radic. Biol. Med. 14 (1993) 303-311.

[9] A.J. Blasco, A.G. Crevillen, M.C. Gonzalez, A. Escarpa, Direct electrochemical sensing and detection of natural antioxidants and antioxidant capacity in vitro systems, Electroanalysis 19 (2007) 2275-2286.

[10] M.F. Barroso, N. de-los-Santos-Alvarez, C. Delerue-Matos, M.B.P.P. Oliveira, Towards a reliable technology for antioxidant capacity and oxidative damage evaluation: electrochemical (bio)sensors, Biosens. Bioelectron. 30 (2011) 1-12.
[11] J. Sochor, J. Dobes, O. Krystofova, B. Ruttkay-Nedecky, P. Babula, M. Pohanka, T. Jurikova, O. Zitka, V. Adam, B. Klejdus, R. Kizek, Electrochemistry as a tool for studying antioxidant properties, Int. J. Electrochem. Sci. 8 (2013) 8464-8489.

[12] S. Chevion, M.A. Roberts, M. Chevion, The use of cyclic voltammetry for the evaluation of antioxidant capacity, Free Radic. Biol. Med. 28 (2000) 860-870.

[13] P.A. Kilmartin, H. Zou, A.L. Waterhouse, A cyclic voltammetry method suitable for characterizing antioxidant properties of wine and wine phenolics, J. Agric. Food Chem. 49 (2001) 1957-1965.

[14] H.D. McBride, D.H. Evans, Rapid voltammetric method for the estimation of tocopherols and antioxidants in oils and fats, Anal. Chem. 45 (1973) 446-449.

[15] A.J. Blasco, M.C. Gonzalez, A. Escarpa, Electrochemical approach for discriminating and measuring predominant flavonoids and phenolic acids using differential pulse voltammetry: towards an electrochemical index of natural antioxidants, Anal. Chim. Acta 511 (2004) 71-81.

[16] M. Seruga, I. Novak, L. Jakobek, Determination of polyphenols content and antioxidant activity of some red wines by differential pulse voltammetry, HPLC and spectrophotometric methods, Food Chem. 124 (2011) 1208-1216.

[17] M.L. Agui, A.J. Reviejo, P. Yanez-Sedeno, J.M. Pingarron, Analytical applications of cylindrical carbon fiber microelectrodes. Simultaneous voltammetric determination of phenolic antioxidants in food, Anal. Chem. 67 (1995) 2195-2200.

[18] A.M.O. Brett, M.E. Ghica, Electrochemical oxidation of quercetin, Electroanalysis 15 (2003) 1745-1750.

[19] S. Chevion, E.M. Berry, N. Kitrossky, R. Kohen, Evaluation of plasma low molecular weight antioxidant capacity by cyclic voltammetry, Free Radic. Biol. Med. 22 (1997) 411-421.

[20] K. Aaby, E. Hvattum, G. Skrede, Analysis of flavonoids and other phenolic compounds using high-performance liquid chromatography with coulometric array detection: Relationship to antioxidant activity, J. Agric. Food Chem. 52 (2004) 4595-4603.

[21] R. Oliveira, J. Marques, F. Bento, D. Geraldo, P. Bettencourt, Reducing antioxidant capacity evaluated by means of controlled potential electrolysis, Electroanalysis 23 (2011) 692-700.

[22] W. Andlauer, J. Héritier, Rapid electrochemical screening of antioxidant capacity (RESAC) of selected tea samples, Food Chem. 125 (2011) 1517-1520.

[23] R. Oliveira, F. Bento, C. Sella, L. Thouin, C. Amatore, Direct electroanalytical method for alternative assessment of global antioxidant capacity using microchannel electrodes, Anal. Chem. 85 (2013) 9057-9063.

[24] P. Tacchini, A. Lesch, A. Neequaye, G. Lagger, J. Liu, F. Cortés-Salazar, H.H. Girault, Electrochemical pseudo-titration of water-soluble antioxidants, Electroanalysis 25 (2013) 922-930.

[25] J.N. Barisci, G.G. Wallace, D. Chattopadhyay, F. Papadimitrakopoulos, R.H. Baughman, Electrochemical properties of single-wall carbon nanotube electrodes, J. Electrochem. Soc. 150 (2003). E409-E15.

[26] R.L. McCreery, Advanced carbon electrode materials for molecular electrochemistry, Chem. Rev. 108 (2008) 2646-2687.

[27] S.K. Vashist, D. Zheng, K. Al-Rubeaan, J.H.T. Luong, F.S. Sheu, Advances in carbon nanotube based electrochemical sensors for bioanalytical applications, Biotech. Adv. 29 (2011) 169-188.

[28] I. Dumitrescu, P.R. Unwin, J.V. MacPherson, Electrochemistry at carbon nanotubes: perspective and issues, Chem. Commun. (2009) 6886-6901.

[29] M. Pumera, Voltammetry of carbon nanotubes and graphenes: excitement, disappointment, and reality, Chem. Rec. 12 (2012) 201-213.

[30] T.S. Miller, N. Ebejer, A.G. Güell, J.V. MacPherson, P.R. Unwin, Electrochemistry at carbon nanotube forests: sidewalls and closed ends allow fast electron transfer, Chem. Commun. 48 (2012) 7435-7437.

[31] C.E. Banks, A. Crossley, C. Salter, S.J. Wilkins, R.G. Compton, Carbon nanotubes contain metal impurities which are responsible for the "Electrocatalysis" seen at some nanotube-modified electrodes, Angew. Chem. Int. Ed. 45 (2006) 25332537.

[32] M.C. Henstridge, L. Shao, G.G. Wildgoose, R.G. Compton, G. Tobias, M.L.H. Green, The electrocatalytic properties of arc-MWCNTs and associated 'carbon onions', Electroanalysis 20 (2008) 498-506.

[33] A. Ambrosi, M. Pumera, Nanographite impurities dominate electrochemistry of carbon nanotubes, Chem. Eur. J. 16 (2010) 10946-10949.

[34] E.J.E. Stuart, M. Pumera, Signal transducers and enzyme cofactors are susceptible to oxidation by nanographite impurities in carbon nanotube materials, Chem. Eur. J. 17 (2011) 5544-5548.

[35] N. Sinha, J. Ma, J.T.W. Yeow, Carbon nanotube-based sensors, J. Nanosci. Nanotechnol. 6 (2006) 573-590.

[36] J.P. Metters, M. Gomez-Mingot, J. Iniesta, R.O. Kadara, C.E. Banks, The fabrication of novel screen printed single-walled carbon nanotube electrodes: electroanalytical applications, Sens. Actuators, B 177 (2013) 1043-1052.

[37] K. Kordas, T. Mustonen, G. Toth, H. Jantunen, M. Lajunen, C. Soldano, S. Talapatra, S. Kar, R. Vajtai, P.M. Ajayan, Inkjet printing of electrically conductive patterns of carbon nanotubes, Small 2 (2006) 1021-1025.

[38] J.W. Song, J. Kim, Y.H. Yoon, B.S. Choi, J.H. Kim, C.S. Han, Inkjet printing of single-walled carbon nanotubes and electrical characterization of the line pattern, Nanotechnology 19 (2008).

[39] J. Zhao, Y. Yu, B. Weng, W. Zhang, A.T. Harris, A.I. Minett, Z. Yue, X.F. Huang, J. Chen, Sensitive and selective dopamine determination in human serum with inkjet printed Nafion/MWCNT chips, Electrochem. Commun. 37 (2013) 32-35.

[40] P. Chen, H. Chen, J. Qiu, C. Zhou, Inkjet printing of single-walled carbon nanotube/ $\mathrm{RuO}_{2}$ nanowire supercapacitors on cloth fabrics and flexible substrates, Nano Res. 3 (2010) 594-603. 
[41] R.P. Tortorich, J.-W. Choi, Inkjet printing of carbon nanotubes, Nanomaterials 3 (2013) 453-468.

[42] J. Delobel, M. Prudent, O. Rubin, D. Crettaz, J.D. Tissot, N. Lion, Subcellular fractionation of stored red blood cells reveals a compartment-based protein carbonylation evolution, J. Proteomics 76 (2012) 181-193.

[43] N. Lion, D. Crettaz, O. Rubin, J.D. Tissot, Stored red blood cells: a changing universe waiting for its map(s), J. Proteomics 73 (2010) 374-385.

[44] M. Prudent, J.D. Tissot, N. Lion, Proteomics of blood and derived products: what's next?, Expert Rev Proteomics 8 (2011) 717-737.

[45] F. Gevi, A. D’Alessandro, S. Rinalducci, L. Zolla, Alterations of red blood cell metabolome during cold liquid storage of erythrocyte concentrates in CPDSAGM, J. Proteomics 76 (2012) 168-180.

[46] M.H. Antonelou, A.G. Kriebardis, K.E. Stamoulis, E. Economou-Petersen, L.H. Margaritis, I.S. Papassideri, Red blood cell aging markers during storage in citrate-phosphate-dextrose-saline-adenine-glucose-mannitol, Transfusion (Paris) 50 (2010) 376-389.

[47] T. Yoshida, S.S. Shevkoplyas, Anaerobic storage of red blood cells, Blood Transfus. 8 (2010) 220-236.

[48] A. D'Alessandro, L. Zolla, Biochemistry of red cell aging in vivo and storage lesions, Hematol. Edu. 7 (2013) 389-396.

[49] A. Tinmouth, I. Chin-Yee, The clinical consequences of the red cell storage lesion, Transfus. Med. Rev. 15 (2001) 91-107.
[50] J. McCullough, Red cell storage: does duration matter?, Blood 121 (2013) 1663-1672

[51] S.B. Solomon, J. Sun, T. Kanias, J. Feng, C.C. Helms, M.A. Solomon, M. Alimchandani, M. Quezado, M.T. Gladwin, D.B. Kim-Shapiro, H.G. Klein, C. Natanson, Mortality increases after massive exchange transfusion with older stored blood in canines with experimental pneumonia, Blood 121 (2013) 1663-1672.

[52] C.G. Koch, L. Li, D.I. Sessler, P. Figueroa, G.A. Hoeltge, T. Mihaljevic, E.H. Blackstone, Duration of red-cell storage and complications after cardiac surgery, N. Engl. J. Med. 358 (2008) 1229-1239.

53] S.R. Goodman, A. Kurdia, L. Ammann, D. Kakhniashvili, O. Daescu, The human red blood cell proteome and interactome, Exp. Biol. Med. 232 (2007) 1391-1408.

[54] A. D'Alessandro, P.G. Righetti, L. Zolla, The red blood cell proteome and interactome: an update, J. Proteome Res. 9 (2010) 144-163.

[55] C.G. Zoski, Ultramicroelectrodes: design, fabrication, and characterization, Electroanalysis 14 (2002) 1041-1051.

[56] C. Amatore, J.M. Saveant, D. Tessier, Charge transfer at partially blocked surfaces. A model for the case of microscopic active and inactive sites, J. Electroanal. Chem. 147 (1983) 39-51.

[57] J.V. Macpherson, C.E. Jones, P.R. Unwin, Radial flow microring electrode: investigation of fast heterogeneous electron-transfer processes, J. Phys. Chem. B 102 (1998) 9891-9897. 